\title{
Higher Order Approximations to Coal Pyrolysis Distribution
}

\author{
Sione Paea ${ }^{1}$ and Mark McGuinness ${ }^{2}$ \\ ${ }^{1}$ School of Computing, Information \& Mathematical Sciences, The \\ University of the South Pacific, Suva, Fiji \\ ${ }^{2}$ School of Mathematics and Statistics, Victoria University of \\ Wellington, New Zealand
}

July 27, 2017

\begin{abstract}
Coal pyrolysis is a complex process involving a large number of chemical reactions. Pyrolysis is a key step in all coal conversion processes. The Distributed Activation Energy Model (DAEM) is a stateof-the art approach to the problem of predicting the amount of volatile released versus activation energy or time. The distribution of mass released is usually assumed to be Gaussian. We present an inverse iterative approach together with a smoothing function to estimate the underlying distribution directly from volatilisation data.
\end{abstract}

\section{Introduction}

Coal pyrolysis has been the subject of numerous studies on reaction kinetics and the amount of total volatile yield. Some of these studies have addressed the individual volatile species and measured the kinetics of species evolution. Modeling coal pyrolysis is important not only for processes where coal is a part of the chemical system, but also for combustion, thermal decomposition, or gasification processes in general.

Howard [1] and Solomon et al. [2] proposed two mathematical models of coal pyrolysis in their research. They describe two models, the Single First Order Reaction model (SFOR) and the more complicated Distributed Activation Energy Model (DAEM). Recent researchers including Niksa and Lau [3], Suuberg [4], Miura and Maki [5] and McGuinness et al. [6] have discussed simplifications of these models. Niksa and Lau [3] claimed that holding the SFOR base-rate constant is a good way to estimate nominal 
rates for any given thermal history. The same devolatilization rate will be predicted as in the DAEM at every instant in the thermal history. By using this approach to explore the relationship between the DAEM and the SFOR model, the activation energy is fixed. Niksa and Lau [3] introduce an effective or nominal rate constant $\langle k\rangle$ which varies with time. They also derive analytical approximations to the DAEM for testing linear or exponential temperature ramping. The resulting rapidly varying double exponential (DExp) function is approximated by a piecewise linear function with three regions. That is, DExp is zero or unity, and the region in between is where it rises linearly from zero to one. This procedure can make the evaluation of the integral much easier where the initial distribution is Gaussian. Therefore it provides an accurate approximation of the full DAEM for all parameters of interest. Parameters which influence the pyrolysis process vary at different stages of the process and depending on the reactor space [7].

Niksa and Lau [3] indicated that this approximate procedure using a piecewise linear function provides a more accurate approximation to the full DAEM for all parameters. This is a refinement of the ideas in Suuberg [4]. Suuberg used a simple step-function approximation to the double exponential ( see also $[1,8,9]$ ), which jumps from zero to one at an energy that varies with time. The use of a Gaussian initial distribution with the step-function gives an error function approximation to the DAEM. This error function will be used later in this study as a foundation function for the development of the inverse problem in the wide distribution case. Niksa and Lau [3] note that some shortcomings remain in using their approximation at lower temperatures, particularly with the numerical solution of the equations for the position of the piecewise linear approximation.

Miura and Maki [5] (see also [10], [11]) consider the inverse problem and present a method to estimate both the distribution $f(E)$ and the rate constant $k_{0}$ from three sets of experiments performed at different heating profiles without assuming any functional forms for $f(E)$ and $k_{0}$. They summarize a procedure with four steps to estimate $f(E)$ and $k_{0}$. For a linearlyramping temperature, they approximate DExp by a step function because DExp changes rather steeply with $E$ at a given temperature. Miura and Maki [5] found the rate of change of volatility with time is proportional to the distribution of volatiles $(f)$. They used this relationship to obtain $f$ from the experimental measurements. McGuinness et al. [6] present a more accurate approximation to the double exponential, which is used in the two cases of narrow and wide distribution. A careful analysis in these two regimes is given based upon asymptotic expansions, leading to systematic methods for rapidly finding accurate approximations.

In this study we review the Simple First Order Reaction Model (SFOR) and Distributed Activation Energy Model (DAEM) in order to understand why the DAEM is a more accurate and appropriate approach to modeling coal pyrolysis. Then we consider how to solve the inverse problem of finding 
the distribution used by the DAEM when the relative width of the initial distribution is much wider than the width of the double exponential term. Finally we focus on a method to reduce the errors due to differencing by first fitting an appropriate smooth function to the data. This appropriate smooth function allows the calculation of higher-order terms, and gives a better fit to data, providing a more accurate estimate of the underlying distribution in the DAEM.

\section{Mathematical Models of Coal Pyrolysis}

\subsection{Existing Models}

The development in this study follows Howard's [1] and Solomon and Hamblen's [12] approaches. The amount and composition of the thermal decomposition products depends on the physicochemical properties of coal and on process parameters. As the process of thermal decomposition of coal evolves, $i$ denotes one particular reaction and coal's constituents are numbered with $i=1 \ldots . n$. The thermal decomposition of coal is assumed to comprise large numbers of independent chemical reactions. Large fragments of the coal molecule are present due to depolymerization and the rupture of various bonds within the coal molecule. The strength of chemical bonds depends on the coal type and rank, related to the occurrence of different reactions at various temperature intervals. The contribution to evolution by a particular reaction is described by a first order equation, so that the rate of pyrolysis is

$$
\frac{\mathrm{d} V_{i}}{\mathrm{~d} t}=k_{i}\left(V_{i}^{*}-V_{i}\right)
$$

where $V_{i}$ is the released mass fraction of volatiles corresponding to the $i$ th constituent, while $V_{i}^{*}$ is the initial mass of constituent $i$ in the coal. The proportionality constant $k_{i}$ is the rate coefficient that is typically associated with temperature by an equation which is Arrhenius in form,

$$
k_{i}=k_{0 i} \exp \left(\frac{-E_{i}}{R T(t)}\right)
$$

where $k_{0 i}$ is the pre-exponential or frequency factor in $\mathrm{sec}^{-1}, E_{i}$ is the apparent activation energy for constituent $i$ in $\mathrm{J} / \mathrm{mol}, R$ is the ideal gas constant in $(\mathrm{J} / \mathrm{mol}$ kelvins) and $T(t)$ is the absolute temperature of the coal particle in Kelvins. Values of $k_{0 i}, E_{i}$, and $V_{i}^{*}$ are estimated from matching with experimental data. Anthony and Howard [13] summarized a collection of experimental rate constant values $k_{i}$, and the associated rate parameters and coal properties. 
The solution to Equation (1) may be written in terms of the mass of volatiles remaining to be released at time $t$ as

$$
\frac{V_{i}^{*}-V_{i}}{V_{i}^{*}}=\exp \left(-\int_{0}^{t} k_{i}(u) d u\right) .
$$

Then the mass of the volatiles released for one sample reaction is

$$
V_{i}=V_{i}^{*}-V_{i}^{*} \exp \left(-\int_{0}^{t} k_{i}(u) d u\right) .
$$

The model developed by Howard [1] and Solomon and Hamblen [12] has been further refined and developed specifically for SFOR and DAEM models which are briefly described in the following.

\subsection{Single First Order Reaction Model (SFOR)}

The simplest method for the description of the kinetics of the pyrolysis reactions is to use a first order reaction for overall weight loss of the volatile and for individual species evolution. The development of the mathematical models above shows that if $i=1$ then the model is referred to as the Single First Order Reaction Model (SFOR). Thus, the rate of pyrolysis is expressed as:

$$
\frac{d V}{d t}=k\left(V^{*}-V\right) .
$$

where the rate constant $k$ is given by:

$$
k=k_{0} \exp \left(\frac{-E}{R T}\right) .
$$

Many authors have approximated the overall process of the complex decomposition and transport phenomena involved in coal pyrolysis. They believed decomposition occurs evenly throughout the volume of the particles as a first order reaction, and its course is determined by the chemical structure of coal [14]. Howard and Essenhigh [15] explained their results by assuming that pyrolysis is a first order reaction with respect to the amount of undecomposed volatile matter. They assume a constant rate which is of the Arrhenius type. Badzioch and Hawksley[16], and Pitt [9] among others, have also shown that the thermal decomposition of coal occurs via first order reactions. The SFOR model is the approach based on holding the activation energy fixed and defining $k$ in Equation (6) as the rate constant which varies with time, $t \rightarrow \infty$. 


\subsection{Distributed Activation Energy Model (DAEM)}

The DAEM is one of the multi-reaction models used widely to clarify the thermal decomposition processes of coal pyrolysis. Pitt [9] assumed that the evolution of a certain substance involves an infinite number of independent chemical reactions by considering a continuous distribution of reactants. That is, many irreversible first-order parallel reactions that have different rate parameters occur simultaneously. In the DAEM model, the dependence on $i$ is replaced by a continuous dependence on activation energy $E$ so the values of $k_{0 i}, E_{i}$ and $V_{i}^{*}$ are not known apriori and must be estimated from the experimental data.

DAEM has been applied to represent the change in overall conversion and the change in the yield of a given component during the coal pyrolysis. The increase in the number of reactions required can cause a problem. This problem is simplified by assuming that the $k_{i}$ 's differ only in activation energy so a common assumption is then to take all the pre-exponential factors, $k_{0 i}$, to have the same value $k_{0}$ for all constituents $i$. Then the number of reactions is large enough to permit the use of a distribution function $f(E)$, acknowledging the differences in the activation energies of the volatiles present. Then $f(E) d E$ represents the fraction of the potential volatile loss $V^{*}$ that have a activation energies between $E$ and $E+d E$. Thus, the total amount of volatile material available for release from the coal in this energy range can be written as:

$$
d V^{*}=V^{*} f(E) d E
$$

with the distribution function $f(E)$ normalized to satisfy

$$
\int_{0}^{\infty} f(E) d E=1
$$

The solution then becomes

$$
\frac{V^{*}-V}{V^{*}}=\int_{0}^{\infty} \exp \left(-\int_{0}^{t} k_{0}(E) e^{-E /(R T(u))} d u\right) f(E) d E .
$$

In DAEM reactions are assumed to consist of set of irreversible first order reactions that have different activation energies and a constant frequency factor. The activation energies are usually assumed to be a Gaussian distribution. The SFOR and DAEM models are discussed in relation to the kinetic expressions for the pyrolysis reaction. These two models are compared in the next section.

\subsection{Comparison of the Two Models}

The SFOR model is used in Howard [1] and Saxena's [17] work. The values of $V^{*}, E$ and $k_{0}$ are determined experimentally. Details of the different 
experimental methods are discussed by Anthony and Howard [13]. The coal is heated so that temperature increases at a constant rate $\frac{d T}{d t}=m$ where $m>0$. Then integrate this uniform heating rate with Equations (5) and (6) in the SFOR model as follows:

$$
\int_{0}^{V} \frac{d V}{V^{*}-V}=\int_{0}^{T} \frac{k_{0}}{m} \exp \left(\frac{-E}{R T}\right) d T .
$$

Since $E / R T \gg 1$ is a good approximation for pyrolysis reactions, the solution to Equation (10) can be approximated as

$$
\frac{V^{*}-V}{V^{*}}=\exp \left(\frac{-k_{0} R T^{2}}{m E} \exp \left(\frac{-E}{R T}\right)\right) .
$$

This approximation applies to the solutions in Equations (3) and (4).

The distribution curve $f(E)$ is generally assumed to be a Gaussian distribution in the DAEM and Equation (9). Within this distribution, the mean activation energy $E_{0}$ and standard deviation $\sigma$ are both determined by the experimental data. Thus

$$
f(E)=\frac{1}{\sigma \sqrt{2 \pi}} \exp \frac{-\left(E-E_{0}\right)^{2}}{2 \sigma^{2}} .
$$

Equations (9) and (12) provide the solution for the DAEM model as follows:

$$
\frac{V^{*}-V}{V^{*}}=\frac{1}{\sigma \sqrt{2 \pi}} \int_{0}^{\infty} \exp \left[-k_{0} \int_{0}^{t} \exp \left(\frac{-E}{R T}\right) d t-\frac{\left(E-E_{0}\right)^{2}}{2 \sigma^{2}}\right] d E .
$$

Equation (13) permits correlation of coal decomposition data using four parameters $\left(V^{*}, E_{0}, \sigma, k_{0}\right)$ and is applicable to a non isothermal process [18] where the distribution is Gaussian.

Comparing the two models, three parameters, $k_{0}, E_{0}$, and $\sigma$ are required in addition to $V^{*}$ for the DAEM model. However for the SFOR model, only two parameters, frequency factor and activation energy are required for analysis. In other words DAEM requires only one additional parameter, $\sigma$, from SFOR model but it is applicable to the description of thermal decomposition processes with different heating rates $[1,19]$. The main drawback of the DAEM model is the computational time required to evaluate the integral, prompting a number of studies of approximations to it.

Niksa and Lau [3] have explored the relationship between the DAEM and the SFOR models with an approach based on holding the activation energy fixed and defining an effective or nominal rate constant $\langle k\rangle$, which varies with time as

$$
\frac{d V}{d t}=\langle k\rangle\left(V^{*}-V\right)
$$


The nominal rates for any given thermal history can be estimated from the SFOR-based rate constant that predicts the same devolatilization rate as the DAEM at every instant in the thermal history. Niksa and Lau [3] note that there is a large variation in $\langle k\rangle$ with time or temperature, and also a more modest variation with coal rank. They also derive analytical approximations to the DAEM for temperatures undergoing linear or exponential ramping. Their approach is based on exploiting the rapid changes occurring in the double exponential (DExp). In Equation (9), the integrand consists of the product of the double exponential term

$$
\mathrm{DExp} \equiv \exp \left(-\int_{0}^{t} k_{0}(E) e^{-E /(R T(u))} d u\right),
$$

and a term representing the distribution of activation energy $f(E)$. Niksa and Lau [3] noted that if $E / R T \gg 1$, and the temperature ramps as $T=$ $m t$, then

$$
\int_{0}^{t} k_{0}(E) e^{-E /(R T(u))} d u \sim \frac{k_{0} R T^{2}}{m E} \exp \left(\frac{-E}{R T}\right) .
$$

Gunes and Gunes [20] discussed the influences of various parameters on the numerical solution of the nonisothermal DAEM Equation (13), while Brown [21] undertook a detailed review of the effect of various parameters on the SFOR model. In the SFOR model, Howard [1] plotted Equation (11) using various activation energies and it clearly illustrated the nature of any parameter changes. The comparison here is focused mainly on the influence of these parameters: heating rate $(m)$, activation energy $(E)$ and the pre-exponential factor $\left(k_{0}\right)$. Both models used various numerical values of parameters to explore the effects of the changes in parameters.

Brown [21] and Gunes and Gunes [20] examined the influence of each parameter on the numerical solution of both models. The first parameter is the influence of heating rate $(m)$ on both models. Their results illustrate that the DAEM and SFOR models show that remaining mass fraction curves are shifted up the temperature scale by an increase in the heating rate. The second parameter is the influence of mean activation energy $\left(E_{0}\right)$. Both models show similar influences. When $E_{0}$ values increased it causes the curves to shift towards the right. By comparing the curves of both models to Howard's [1] curve of data on the total yield of volatiles, it seems that the DAEM curve gives a more realistic result, due to the influence of standard deviation $(\sigma)$. The third parameter is the pre-exponential factor $\left(k_{0}\right)$. The DAEM and the SFOR models show that an increase in $k_{0}$ value causes the curves to shift toward the left. The effect of each of the three parameters is to cause the curves to shift up the temperature scale.

A model such as the Single First-Order Reaction model (SFOR) is strictly applicable only to homogeneous systems in which decomposition from the 
source $V^{*}$ is due to a single chemical process which occurs with a single activation energy $E$. Solomon et al. [19] has shown that for the devolatilization of coal, the values of $k_{0}$ and $E$ determined for one heating rate are not appropriate when used for another heating rate. Some other simple models were used also and, like the single first order model, they were applicable only under limited experimental conditions [1]. Howard [1] plotted Equation (9) using what might be called reasonable parameters in fitting the data to the total yield of volatile. The resulting graph showed Howard some inadequacies in the single reaction model. In an attempt to improve the SFOR model, Howard specified that the activation energy and pre-exponential factor must be very low to approximately fit the temperature dependence that results from the occurrence of different reactions at different temperature intervals. However, he still concluded that the SFOR model was inadequate for dealing with the complexities of coal pyrolysis.

When modeling industrial processes where large particles or lumps of coal are involved, heat transfer cannot be neglected and the change of temperature cannot be described with one uniform heating rate. In general, the change of temperature in solids is modelled by the transport partial differential equation, which may be nonlinear. In other words more complicated reactions like coal pyrolysis cannot be adequately modelled by a single reaction, due to large variations in the value of $k_{0}$ and $E_{0}$ with the heating rate $\frac{d T}{d t}[19]$. Therefore, researchers realized that the SFOR model could only be applied to limited experimental conditions and that they needed a model which could be applied to more complex experimental conditions of coal pyrolysis. They then moved to a more complicated model such as the Distributed Activation Energy Model (DAEM) model. It is adapted from Vand's [8] treatment of independent parallel processes in modeling the resistance of metallic films. DAEM has proved very successful in describing the pyrolysis of various coal types under differing temperature histories.

The DAEM can also be applied to explain the thermal decomposition processes of the pyrolysis of coal and other materials, including biomass, residual oils, resin chars [22] and kerogen [23]. Anthony et al. [18] and Howard [1] described the DAEM as applicable over a wide range of thermal conditions. The model was originally developed to predict volatile yields during rapid pyrolysis of coal but has also been applied at the relatively low heating rate encountered during thermal decomposition of coal to coke (Merrick, [24]). DAEM is the simplest model that depicts devolatilization rates during transient heating over a broad range of heating rates. It is also the only formalism in devolatilization modeling that captures the observed density of reaction time scales for this process [1]. 


\subsection{Evaluation of the Two Models}

The two models have their shortcomings for application to the pyrolysis of coal. According to Howard [1], the most serious problem of Equation (5) and (6) in the SFOR model is the apparently asymptotic yield of volatiles that is observed after some time at the final temperature. As a result, the apparent value of $V^{*}$ as a function of final temperature is mechanistically inconsistent with the equations and is mathematically unamenable. Howard also plotted Arrhenius plots for rate constants from the work of different researchers and labelled the different graphs using time zones in some of the correlations. The relatively slow rate of weight loss observed after extended times at a given temperature requires a set of parameter values that differ markedly from those that fit the behavior of the graph over short time. Howard [1] clearly stated that coal pyrolysis is not a single reaction, but rather a multiplicity of overlapping decompositions concentrated in different time intervals for isothermal pyrolysis, or in different time and temperature intervals for the usual case of pyrolysis during heatup. He concluded that any one set of parameter values for these equations cannot be expected to represent data accurately over a wide range of conditions. The SFOR model could only be applied in limited conditions and in this way was problematic.

The problems were then solved by applying the DAEM model to these limited conditions. However, the main difficulty with the DAEM solution is a complicated double integral which can require significant computing resources, particularly when it needs evaluating many times [25]. Miura [10] also discussed in his work that the DAEM model has two major weak points. The first is the assumption of a constant $k_{0}$ value for all reactions. The other is the assignment of the Gaussian distribution to $f(E)$. It is possible to estimate $f(E)$ from experimental data without assuming the Gaussian distribution as performed by Vand [8]. However, in order to use the Gaussian distribution, a constant value must be assigned to $k_{0}$ beforehand in order to estimate $f(E)$. Miura [10] presented a simple method to overcome this problem. The simple method was applied to estimate $f(E)$ and $k_{0}(E)$ from three sets of experimental data without any assumption on the functional form of $f(E)$ or $k_{0}$ for the pyrolysis of three kinds of coal.

In all of the recent research, the DAEM has been described as a more powerful model for evaluating the complex experimental conditions of coal pyrolysis. Researchers show that the shortcomings of the SFOR model can be solved by the use of the DAEM. The DAEM is generally recognized to be the most appropriate approach to model coal pyrolysis.

\section{Mathematical Formulation}

In Equation (9) for the DAEM, the integrand consists of the product of two major parts. First, the double exponential (DExp) term is determined by 
the temperature during the experiment and depends on time through the temperature history experienced by the sample. The other term representing the initial distribution $f(E)$ is determined by the type of coal being considered and it is independent of time, and depends on the distribution of volatiles in the sample. So Equation (9) can be re-written as

$$
v=\int_{0}^{\infty}(\operatorname{DExp})(f(E)) d E,
$$

where $v=1-\left(V / V^{*}\right)$ is the fraction of the volatile yield not yet released. Another way of writing the double integral in Equations (9) and (16) is in the form

$$
v=\int_{0}^{\infty} \exp \left(-\int_{0}^{t} k_{0}(E) e^{-E /(R T(u))} d u+\ln (f(E))\right) d E
$$

The effect of constant temperature $T(u)=T_{0}$ on DExp is discussed first. It is followed by an investigation of ramping temperature histories.

\subsection{Double exponential integrand simplification for DAEM}

McGuinness et al. [6] developed an approximation to DExp where $T(u)$ is specified and $E$ can take any positive value. Their approach was similar to the work presented by Niksa and Lau [3] but uses more systematic methods and a more accurate approximation. The approach taken is similar to that of Niksa and Lau [3] and McGuinness et al. [6] but it is different in the sense that here we use higher-order terms. The more such terms we provide, the better the underlying distribution in the DAEM. The equation of the double exponential term is shown as

$$
\mathrm{DExp} \equiv \exp \left(-\int_{0}^{t} k_{0} e^{-E /(R T(u))} d u\right) .
$$

The integral of DExp is particularly simple when temperature is constant, $T(u)=T_{0}$. Equation (17) is integrated with respect to the dummy variable $u$ from zero to $t$ to obtain

$$
\mathrm{DExp} \equiv \exp \left(-t k_{0} e^{-E / R T_{0}}\right) .
$$

In order to apply the systematic simplifications of this integrand it is necessary to assume some typical range of the parameters and functions on which it depends. The frequency factors are typically in the range of $10^{10} \leq k_{0} \leq 10^{13} \mathrm{~s}^{-1}$, whereas the activation energies fall into the region of $100-300 \mathrm{~kJ} \mathrm{~mol}^{-1}$. The temperature history depends on the particular experiments but $100-600^{\circ} \mathrm{C}$ is mainly used in pyrolysis. However, we can also apply this DAEM model of the interest in combustion problem where the 
temperature range can be significantly larger than any other application. When both of the parameters $E / R T_{0} \sim 10$ and $t k_{0} \sim 10^{10}$ from Equation (18) are given large values, the DExp function changes rather steeply with $E$. In order to describe the stepwise function simplification of Gaussian distribution, the case of ramping temperature with $T=m t$ is used in Equation (17) gives

$$
\mathrm{DExp} \equiv \exp \left(-\int_{0}^{t} k_{0} e^{-E / R m u} d u\right) .
$$

In Equation (19), the integral inside the exponential function can be approximated using the conventional Laplace transform approach. The parameter $E /(R m t)$ is assumed to be large. Evaluating the integral of the DExp function produces the following well known asymptotic solution,

$$
\exp \left(-\int_{0}^{t} k_{0} e^{-E / R m u} d u\right) \sim \exp \left(-\frac{k_{0} R m t^{2}}{E} e^{-E / R m t}\right), \quad \frac{E}{R m t} \rightarrow \infty .
$$

Equations (20) and (15) have the same form as Equation (11) when $T=$ $m t$. These equations are exactly the same as the equation resulting from the p-function presented by Miura [10]. Equation (20) can be rewritten as:

$$
\exp \left(-\exp \left(\frac{E_{s}-E}{E_{w}}\right)\right)
$$

and as noted above, when $E$ is increased over a range of size $E_{w}$ around $E_{s}$, the function changes rapidly from zero to one. To obtain the desired form, we let

$$
g(E)=\frac{E_{s}-E}{E_{w}}
$$

and we set

$$
\exp \left(-\frac{k_{0} R m t^{2}}{E} e^{-E / R m t}\right)=\exp (-\exp (g(E))),
$$

which implies that

$$
g(E)=-\frac{E}{R m t}+\ln \left(\frac{k_{0} R m t^{2}}{E}\right) .
$$

Since only the behavior near $E_{s}$ is of interest, this function is expanded in a Taylor series as follows in order to allow the terms $E_{s}$ and $E_{w}$ to be identified:

$$
\begin{aligned}
g(E) \sim & g\left(E_{s}\right)+\left(E-E_{s}\right) g^{\prime}\left(E_{s}\right)+\left(E-E_{s}\right)^{2} g^{\prime \prime}\left(E_{s}\right) / 2+ \\
& \left(E-E_{s}\right)^{3} g^{\prime \prime \prime}\left(E_{s}\right) / 6+\ldots
\end{aligned}
$$


Comparing Equation (21) and the definition of $g(E)$ gives the values of $g\left(E_{s}\right)=0$ and $g^{\prime}\left(E_{s}\right)=-1 / E_{w}$, hence:

$$
g\left(E_{s}\right) \equiv-\frac{E_{s}}{R m t}+\ln \left(\frac{k_{0} R m t^{2}}{E_{s}}\right)=0
$$

and

$$
g^{\prime}\left(E_{s}\right) \equiv-\frac{1}{R m t}-\frac{1}{E_{s}}=-\frac{1}{E_{w}} .
$$

Solving and simplifying Equation (22) and (23) gives the solutions $E_{s}=$ $R m t Y\left(k_{0} t\right)$ and $E_{w}=\frac{R m t E_{s}}{R m t+E_{s}}$ where $Y(x)$ is the LambertW function considered to be the one real root of the equation

$$
Y e^{Y}=x .
$$

Writing Equation (22) in the form of Equation (24) produces

$$
\frac{E_{s}}{R m t} e^{E_{s} /(R m t)}=k_{0} t
$$

The LambertW function $Y(x)$ is used often in this study in order to develop the formula of the inverse problem. So it is useful to understand approximations to $Y(x)$ for small and large $x$ corresponding to short and long time periods [26].

$$
Y \sim x-x^{2}, \quad x \ll 1,
$$

and

$$
Y \sim \ln \left(\frac{x}{\ln \left(\frac{x}{\ln x}\right)}\right), \quad x \gg 1 .
$$

In Equation (9), the total integrand is the product of the double exponential function (DExp) and initial distribution $(f(E))$. The DExp has been described as a smooth step-function which changes rapidly from zero to one. This rapid change is due to the large size of $t k_{0}$ in a range of activation energies of width $E_{w}$ around the value $E=E_{s}$, with $E_{s}$ and $E_{w}$ varying with time. The $f(E)$ is presumed to be a Gaussian distribution with $\sigma$ as the standard deviation of the distribution and $E_{0}$ as the mean activation energy. Both parameters are treated as constant values. There are two different limits to evaluate the Gaussian distribution: wide initial distribution and narrow initial distribution.

This study focuses on the wide distribution case, where the initial distribution $f(E)$ is wider than the width $E_{w}$. As time progresses, Dexp moves across the distribution, bringing the integrand from zero times the distribution, to one times the distribution. Thus the initial distribution is progressively chopped off from the left by step-like function. The location of the maximum of the total integrand can move significantly, and the shape 
becomes quite skewed. This behaviour of the integrand is know to originate in the nature of DExp when it is approximated by a step-function (see also $[1],[4],[8],[9])$.

In order to describe the approach, the initial distribution $(f(E))$ is taken to be a Gaussian distribution with mean activation energy $E_{0}$ and standard deviation $\sigma$. Approximation is sought to the integral

$$
v=\frac{1}{\sigma \sqrt{2 \pi}} \int_{0}^{\infty} \exp (h(E)) d E
$$

where

$$
h(E)=-\exp \left(\frac{E_{s}-E}{E_{w}}\right)-\frac{\left(E-E_{0}\right)^{2}}{2 \sigma^{2}}
$$

and $E_{s}$ and $E_{w}$ are functions of time as stated earlier. The energy is rescaled as $y=E / E_{0}$, so the problem becomes

$$
v=\sqrt{\frac{\alpha}{\pi}} \int_{0}^{\infty} \exp (h(y)) d y
$$

where

$$
h(y)=-\exp \left(\frac{y_{s}-y}{y_{w}}\right)-\alpha(y-1)^{2}
$$

where $y_{s}=\frac{E_{s}}{E_{0}}, y_{w}=\frac{E_{w}}{E_{0}}$, and $\alpha=\frac{1}{2}\left(\frac{E_{0}}{\sigma}\right)^{2}$. Note that in practice $\alpha \gg 1$. Consider our two special cases of temperature history in terms of the rescaled parameters $y$ and $\tau=k_{0} t$ :

1. For a constant temperature where $T=T_{0}$,

$$
y_{s}=\frac{R T_{0}}{E_{0}} \ln \tau, \quad y_{w}=\frac{R T_{0}}{E_{0}} .
$$

2. For a linear ramping temperature where $T=m t$,

$$
y_{s}=\frac{R m \tau}{k_{0} E_{0}} Y(\tau), \quad y_{w}=\frac{y_{s}}{1+Y(\tau)} .
$$

These equations are used to approximate $v$, in the case of a linear ramping temperature, in the next section, when the initial distribution is much wider than DExp.

\section{The Wide Distribution Case}

The following sections concentrate on the case where the initial distribution $(f(E))$ is much wider than the double exponential (DExp). As discussed above, the DExp is approximated as a smoothed step-function. It rises 
rapidly from zero to one in a range of activation energies of width $E_{w}$ around the value $E=E_{s}$, where $E_{s}$ and $E_{w}$ vary with time. We consider the limit $y_{w} \sqrt{\alpha} \ll 1$. The step-function $U$ is defined to be

$$
U\left(y-y_{s}\right)= \begin{cases}0 & , \text { if } y<y_{s} \\ 1 & , \text { if } y \geq y_{s}\end{cases}
$$

Equation (25) can be written in the form

$$
\begin{aligned}
v= & \sqrt{\frac{\alpha}{\pi}} \int_{0}^{\infty}\left[\exp \left(-\exp \left(\frac{y_{s}-y}{y_{w}}\right)\right)-U\left(y-y_{s}\right)\right] \exp \left(-\alpha(y-1)^{2}\right) d y+ \\
& \sqrt{\frac{\alpha}{\pi}} \int_{y_{s}}^{\infty} \exp \left(-\alpha(y-1)^{2}\right) d y .
\end{aligned}
$$

The first integrand in Equation (27) is the product of the initial distribution and a function which is very small everywhere except in a neighbourhood of size $y_{w}$ around the point $y=y_{s}$. Hence the initial distribution term is expanded in a Taylor series about $y=y_{s}$,

$$
\begin{aligned}
& \sqrt{\frac{\alpha}{\pi}} \int_{0}^{\infty}\left[\exp \left(-\exp \left(\frac{y_{s}-y}{y_{w}}\right)\right)-U\left(y-y_{s}\right)\right] \exp \left(-\alpha(y-1)^{2}\right) d y= \\
& \sqrt{\frac{\alpha}{\pi}} \int_{0}^{\infty}\left[\exp \left(-\exp \left(\frac{y_{s}-y}{y_{w}}\right)\right)-U\left(y-y_{s}\right)\right]\left(1-\left(y-y_{s}\right)\right. \\
& \left.2 \alpha\left(y_{s}-1\right)+\ldots\right) \exp \left(-\alpha\left(y_{s}-1\right)^{2}\right) d y .
\end{aligned}
$$

The right hand side of Equation (28) can then be approximated as

$$
\sqrt{\frac{\alpha}{\pi}} y_{w} e^{-\alpha\left(y_{s}-1\right)^{2}} \int_{-\infty}^{\infty}\left[e^{-e^{-x}}-U(x)\right]\left(1-\left(y-y_{s}\right) 2 \alpha\left(y_{s}-1\right)+\ldots\right) d x,
$$

so that

$$
\begin{aligned}
& \sqrt{\frac{\alpha}{\pi}} \int_{0}^{\infty}\left[\exp \left(-\exp \left(\frac{y_{s}-y}{y_{w}}\right)\right)-U\left(y-y_{s}\right)\right] \exp \left(-\alpha(y-1)^{2}\right) d y= \\
& \quad \sqrt{\frac{\alpha}{\pi}} y_{w} e^{-\alpha\left(y_{s}-1\right)^{2}}\left[b_{0}-2 \alpha y_{w}\left(y_{s}-1\right) b_{1}+\alpha y_{w}^{2}\left\{2 \alpha\left(y_{s}-1\right)^{2}-1\right\} b_{2}\right. \\
& \left.\quad+\frac{2}{3} y_{w}^{3} \alpha^{2}\left\{2\left(y_{s}-1\right)+2 \alpha\left(y_{s}-1\right)^{3}+1\right\} b_{3}+\ldots\right],
\end{aligned}
$$

where

$$
b_{i} \equiv \int_{-\infty}^{\infty} x^{i}\left(e^{-e^{-x}}-U(x)\right) d x \quad i=0,1,2, \ldots
$$

remain to be evaluated. The values of $b_{i}$ need to be calculated once, as they are independent of any parameters.

$$
b_{0} \approx-0.5772, b_{1} \approx-0.98906, b_{2} \approx-1.81496, b_{3} \approx-5.89037 .
$$


Most of the previous simplifications of the step-function approximations used only the conventional error function as a dominant function and ignored the first integral. The case of a Gaussian initial distribution in the step-function gives an error function approximation to the DAEM illustrated in the second integral of Equation 27. Then noting that

$$
\sqrt{\frac{\alpha}{\pi}} \int_{y_{s}}^{\infty} \exp \left(-\alpha(y-1)^{2}\right) d y=\frac{1}{2} \operatorname{erfc}\left(\sqrt{\alpha}\left(y_{s}-1\right)\right),
$$

where erfc is the complementary error function

$$
\operatorname{erfc}\left(\sqrt{\alpha}\left(y_{s}-1\right)\right)=\frac{1}{\sqrt{\pi}} \int_{\sqrt{\alpha}\left(y_{s}-1\right)}^{\infty} e^{-u^{2}} d u
$$

combining these results gives an approximation to the solution of Equation (27).

$$
\begin{aligned}
v \sim & \frac{1}{2} \operatorname{erfc}\left(\sqrt{\alpha}\left(y_{s}-1\right)\right)+\sqrt{\frac{\alpha}{\pi}} y_{w} e^{-\alpha\left(y_{s}-1\right)^{2}}\left[b_{0}+2 \alpha y_{w}\left(y_{s}-1\right) b_{1}+\alpha y_{w}^{2}\right. \\
& \left.\left\{2 \alpha\left(y_{s}-1\right)^{2}-1\right\} b_{2}+\frac{2}{3} y_{w}^{3} \alpha^{2}\left\{2\left(y_{s}-1\right)+2 \alpha\left(y_{s}-1\right)^{3}+1\right\} b_{3}\right]
\end{aligned}
$$

This expansion is only valid when $\alpha y_{w}\left(y_{s}-1\right) \ll 1$ and in the limit $y_{w} \sqrt{\alpha} \rightarrow$ 0 , which apply when the initial distribution $f(E)$ is much wider than the width $y_{w}$ of DExp. Then each successive term on the right-hand side of Equation. (29) is smaller than the previous term. This asymptotic approach, illustrated above for the case that the distribution of volatiles is Gaussian in the energy, generalises to gives the following result for a general underlying distribution $f(E)$ that is not necessarily Gaussian:

$$
\begin{aligned}
v \sim & \int_{y s}^{\infty} f(y) d y+y_{w} b_{0} f\left(y_{s}\right)+y_{w}^{2} b_{1} f^{\prime}\left(y_{s}\right)+y_{w}^{3} b_{2} f^{\prime \prime}\left(y_{s}\right) / 2+ \\
& y_{w}^{4} b_{3} f^{\prime \prime \prime}\left(y_{s}\right) / 6+y_{w}^{5} b_{4} f^{i v}\left(y_{s}\right) / 24+y_{w}^{6} b_{5} f^{v}\left(y_{s}\right) / 120 .
\end{aligned}
$$

Equation (30) is now used to solve the inverse problem of finding the distribution $f$ given data for $v$, in the case of a relatively wide distribution.

\subsection{Inverse Problem}

The rate of volatilization from the general result in Equation (30) is rewritten in non-dimensional form that gives a procedure for considering the inverse problem with greater accuracy.

$$
\begin{aligned}
\frac{d v}{d \tau} \sim & {\left[-f\left(y_{s}\right)+y_{w} b_{0} f^{\prime}\left(y_{s}\right)+y_{w}^{2} b_{1} f^{\prime \prime}\left(y_{s}\right)+\frac{1}{2} y_{w}^{3} b_{2} f^{\prime \prime \prime}\left(y_{s}\right)+\right.} \\
& \left.\frac{1}{6} y_{w}^{4} b_{3} f^{i v}\left(y_{s}\right)\right] \frac{d y_{s}}{d \tau}+\left[b_{0} f\left(y_{s}\right)+2 b_{1} y_{w} f^{\prime}\left(y_{s}\right)+\frac{3}{2} y_{w}^{2} b_{2} f^{\prime \prime}\left(y_{s}\right)+\right. \\
& \left.\frac{2}{3} y_{w}^{3} b_{3} f^{\prime \prime \prime}\left(y_{s}\right)+\frac{5}{24} y_{w}^{4} b_{4} f^{i v}\left(y_{s}\right)\right] \frac{d y_{w}}{d \tau}
\end{aligned}
$$


Since $E_{0}$ is unknown for the inverse problem, it is convenient to write Equation (31) in dimensional form:

$$
\begin{aligned}
\frac{d v}{d t} \sim & \left(-\frac{d E_{s}}{d t}+b_{0} \frac{d E_{w}}{d t}\right) f\left(E_{s}\right)+\left(b_{0} E_{w} \frac{d E_{s}}{d t}+2 b_{1} E_{w} \frac{d E_{w}}{d t}\right) f^{\prime}\left(E_{s}\right)+ \\
& \left(b_{1} E_{w}^{2} \frac{d E_{s}}{d t}+\frac{3}{2} b_{2} E_{w}^{2} \frac{d E_{w}}{d t}\right) f^{\prime \prime}\left(E_{s}\right)+\left(\frac{1}{2} b_{2} E_{w}^{3} \frac{d E_{s}}{d t}+\frac{2}{3} b_{3} E_{w}^{3} \frac{d E_{w}}{d t}\right) \\
& f^{\prime \prime \prime}\left(E_{s}\right)+\left(\frac{1}{6} b_{3} E_{w}^{4} \frac{d E_{s}}{d t} s+\frac{5}{24} b_{4} E_{w}^{4} \frac{d E_{w}}{d t}\right) f^{i v}\left(E_{s}\right) .
\end{aligned}
$$

Note that $f\left(E_{s}\right)$ is to be determined whereas $E_{s}$ and $E_{w}$ are known functions depending on $t$ (provided that $k_{0}$ is known). Niksa and Lau [3] discussed replacing the dependence on $t$ with dependence on $E_{s}$, by inverting $E_{s}(t)$.

Our approach is to expand $f$ as a power series in $\epsilon, f \sim f_{0}+\epsilon f_{1}+\ldots$. , in the previous equation. We leverage the relative narrowness of Dexp (and define $\epsilon$ ) by putting $E_{w}=\epsilon e_{w}$, where $\epsilon \ll 1$ and $e_{w}$ is of order one. The rate of volatilisation then becomes

$$
\begin{aligned}
\frac{d v}{d t} \sim & -\frac{d E_{s}}{d t} f_{0}-\epsilon \frac{d E_{s}}{d t} f_{1}-\epsilon^{2} \frac{d E_{s}}{d t} f_{2}-\epsilon^{3} \frac{d E_{s}}{d t} f_{3}-\cdots+\epsilon b_{0} \frac{d e_{w}}{d t} f_{0}+ \\
& \epsilon^{2} b_{0} \frac{d e_{w}}{d t} f_{1}+\epsilon^{3} b_{0} \frac{d e_{w}}{d t} f_{2}+\cdots+\epsilon e_{w} b_{0} \frac{d E_{s}}{d t} f_{0}^{\prime}+\epsilon^{2} e_{w} b_{0} \frac{d E_{s}}{d t} f_{1}^{\prime}+ \\
& \epsilon^{3} e_{w} b_{0} \frac{d E_{s}}{d t} f_{2}^{\prime}+\cdots+2 \epsilon^{2} e_{w} b_{1} \frac{d e_{w}}{d t} f_{0}^{\prime}+2 \epsilon^{3} e_{w} b_{1} \frac{d e_{w}}{d t} f_{1}^{\prime}+\cdots+ \\
& \epsilon^{2} e_{w}^{2} b_{1} \frac{d E_{s}}{d t} f_{0}^{\prime \prime}+\epsilon^{3} e_{w}^{2} b_{1} \frac{d E_{s}}{d t} f_{1}^{\prime \prime}+\cdots+\frac{3}{2} \epsilon^{3} e_{w}^{2} b_{2} \frac{d e_{w}}{d t} f_{0}^{\prime \prime}+\cdots+ \\
& \frac{1}{2} \epsilon^{3} e_{w}^{3} b_{2} \frac{d E_{s}}{d t} f_{0}^{\prime \prime \prime}+\ldots
\end{aligned}
$$

The boundary conditions $f \rightarrow 0$ when $E_{s} \rightarrow 0$ or $\infty$ are satisfied by the zeroth-order solution and regular series expansion techniques provide a way to approximate $f$ - coefficients of powers of $\epsilon$ in Equation (33) are equated to obtain a series of equations for the terms in the expansion of $f$. The first result is the leading-order term

$$
f_{0}=-\frac{d v / d t}{d E_{s} / d t},
$$

which provides an estimate of the underlying distribution. The result obtained by adding to the leading-order term $f_{0}$, the higher-order correction term

$$
\epsilon f_{1}=A_{0}\left(E_{w} \frac{d f_{0}}{d E_{s}}+\frac{\frac{d E_{w}}{d t} f_{0}}{\frac{d E_{s}}{d t}}\right),
$$

which is non Gaussian, does provide a more accurate estimate of the underlying distribution in the DAEM than the leading-order itself. We further 
improved approximation by calculating the higher-order terms $\epsilon^{2} f_{2}$ and $\epsilon^{3} f_{3}$ shown below:

$$
\begin{aligned}
\epsilon^{2} f_{2}= & \epsilon A_{0}\left(\frac{\frac{d E_{w}}{d t} f_{1}}{\frac{d E_{s}}{d t}}+E_{w} f_{1}^{\prime}\right)+A_{1} E_{w}\left(2 \frac{\frac{d E_{w}}{d t} f_{0}^{\prime}}{\frac{d E_{s}}{d t}}+E_{w} f_{0}^{\prime \prime}\right) \\
\epsilon^{3} f_{3}= & \epsilon^{2} A_{0}\left(\frac{\frac{d E_{w}}{d t} f_{2}}{\frac{d E_{s}}{d t}}+f_{2}^{\prime}\right)+\epsilon A_{1} E_{w}\left(2 \frac{\frac{d E_{w}}{d t} f_{1}^{\prime}}{\frac{d E_{s}}{d t}}+E_{w} f_{1}^{\prime \prime}\right)+ \\
& \frac{1}{2} E_{w}^{2} A_{2}\left(3 \frac{\frac{d E_{w}}{d t} f_{0}^{\prime \prime}}{\frac{d E_{s}}{d t}}+E_{w} f_{0}^{\prime \prime \prime}\right) .
\end{aligned}
$$

Each improvement requires higher-order derivatives (differences) to be calculated, and without some form of prior smoothing (data fitting) this leads to data errors that become too large.

\section{$5 \quad$ Data Fitting}

In this section we indicate an improved method for solving the inverse problem, that avoids the numerical errors that arise from repeatedly differencing volatilisation data as in the previous section. The key idea is to firstly use an iterative method to fit the leading order error function solution based on a Gaussian distribution to data. Then this leading order approximation can be regarded as a particularly appropriate smoothing function for the data, that can be used to provide derivatives that are less prone to numerical error due to truncation or measurement error. Note that although we start with a first approximation based on a Gaussian distribution, we do not require the underlying distribution to remain Gaussian further along the refinement path.

We consider Eqn. (30) as providing a succession of approximations to $v$,

$$
v \sim v^{(1)}+v^{(2)}+\ldots
$$

so that in the case of $f$ approximated by a Gaussian distribution $f_{0}$,

$$
v^{(1)}=\int_{y s}^{\infty} f(y) d y
$$

and we approximate this with

$$
v_{0}^{(1)}\left(t, E_{0}, \sigma, k_{0}\right)=\int_{y s}^{\infty} f_{0}(y) d y=\frac{1}{2} \operatorname{erfc}\left(\sqrt{\alpha}\left(y_{s}-1\right)\right)
$$

where

$$
\begin{aligned}
\alpha & =\frac{1}{2} \frac{E_{0}{ }^{2}}{\sigma^{2}}, \\
E_{s} & =\operatorname{Rmt} Y\left(k_{0} t\right), \\
y_{s} & =\frac{E_{s}}{E_{0}},
\end{aligned}
$$


and $Y$ is the LambertW function. We fit $v_{0}^{(1)}$ to data using the LevenburgMarquardt method (Seber and Wild [27]), which is a robust combination of the Gauss-Newton method and the method of steepest descents. A Jacobian matrix is computed from the partial derivatives of $v^{(1)}$,

$$
\begin{gathered}
\frac{\partial v^{(1)}}{\partial E_{0}}=\frac{1}{\sqrt{2 \pi} \sigma} \exp \left[-\frac{\left(R m t Y\left(k_{0} t\right)-E_{0}\right)^{2}}{2 \sigma^{2}}\right], \\
\frac{\partial v^{(1)}}{\partial \sigma}=\frac{1}{\sqrt{2 \pi} \sigma^{2}} \exp \left[-\frac{\left(R m t Y\left(k_{0} t\right)-E_{0}\right)^{2}}{2 \sigma^{2}}\right]\left(\frac{\operatorname{Rmt} Y\left(k_{0} t\right)}{E_{0}}-1\right),
\end{gathered}
$$

and

$$
\frac{\partial v^{(1)}}{\partial k_{0}}=-\frac{1}{\sqrt{2 \pi} \sigma} \exp \left[-\frac{\left(\operatorname{Rmt} Y\left(k_{0} t\right)-E_{0}\right)^{2}}{2 \sigma^{2}}\right]\left(\frac{\operatorname{Rmt} Y\left(k_{0} t\right)}{k_{0}\left(1+Y\left(k_{0} t\right)\right)}\right)
$$

\subsection{Data Generated with a Gaussian}

The solution $v^{(1)}$ is used to generate ersatz data, by evaluating it on a mesh of values of time, and adding $\pm 5 \%$ random error to each data point. Parameter values used to generate the data are $m=650 \mathrm{~K} / \mathrm{s}$ and $R=8.3144 \mathrm{~J} /(\mathrm{mol}$ kelvins). Then we used the Levenburg-Marquardt method, to try to recover the correct parameter values $E_{0}, \sigma$ and $k_{0}$ by fitting to the data. The shape of $v^{(1)}$ depends on $E_{0}$ and $k_{0}$ in a way that is highly correlated, so that fitting $v^{(1)}$ using a simple Gauss-Newton method tends to fail to converge. The initial parameter estimates chosen to begin the numerical fitting procedure are: $E_{0}=2.20 \mathrm{E} 5 \mathrm{~J} / \mathrm{mol}, \sigma=44 \mathrm{E} 3 \mathrm{~J} / \mathrm{mol}$ and $k_{0}=1.33 \mathrm{E} 10 \mathrm{~s}^{-1}$. The results after each iteration, that is, the parameter values obtained by fitting to the data at each step of the method, are shown in Table 1.

\begin{tabular}{|c|c|c|c|c|}
\hline $\boldsymbol{\theta}^{(n)}$ & $E_{n}$ & $\sigma_{n}$ & $k_{n}$ & $S\left(\theta^{(n)}\right)$ \\
\hline$\theta^{(0)}$ & $0.220 \mathrm{E} 6$ & $0.44 \mathrm{E} 5$ & $1.33 \mathrm{E} 10$ & 0.1204338066 \\
$\theta^{(1)}$ & 211035.201 & 46249.9895 & $2.47972118 \mathrm{E} 10$ & 0.01237329944 \\
$\theta^{(2)}$ & 210310.815 & 42077.4605 & $2.99977767 \mathrm{E} 10$ & 0.006463076506 \\
$\theta^{(3)}$ & 212992.377 & 44346.2851 & $5.45149898 \mathrm{E} 10$ & 0.009266540071 \\
$\theta^{(4)}$ & 214391.234 & 42993.6278 & $4.64972779 \mathrm{E} 10$ & 0.006461018969 \\
$\theta^{(5)}$ & 214344.064 & 42968.0639 & $4.70126252 \mathrm{E} 10$ & 0.006452457778 \\
\hline
\end{tabular}

Table 1: The results from $E_{0}=2.20 \mathrm{E} 5 \mathrm{~J} / \mathrm{mol}, \sigma=44 \mathrm{E} 3 \mathrm{~J} / \mathrm{mol}$ and $k_{0}=$ $1.33 \mathrm{E} 10 \mathrm{~s}^{-1}$.

The parameters in Table 1 illustrate that the first two iterations gave better estimates for each parameter and caused a decrease in the sum of squares $S$. The conditioning factor for those iterations was divided by a factor of 10, reflecting that the Levenberg-Marquardt direction was chosen to move in the direction of the Gauss-Newton method. However the third iteration shows the sum of squares started to increase in value. The response 
of the Levenberg-Marquardt algorithm was then to increase the conditioning factor by multiplying by a factor of 10 to push the algorithm in the direction corresponding using that indicated by the method of steepest descents. The Levenberg-Marquardt iterative method is repeated, with the sum of squares error terms $S$ reducing until the results satisfy Equation (42) below

$$
\frac{S_{\text {old }}-S_{\text {new }}}{S_{\text {new }}} \leq 10^{-5} .
$$

At this point the Levenberg-Marquardt algorithm was taken to have converged. The resulting fit is plotted for each iteration starting with the starting function, and shown in Figure 1. In Figure 1 the later iterations lie close

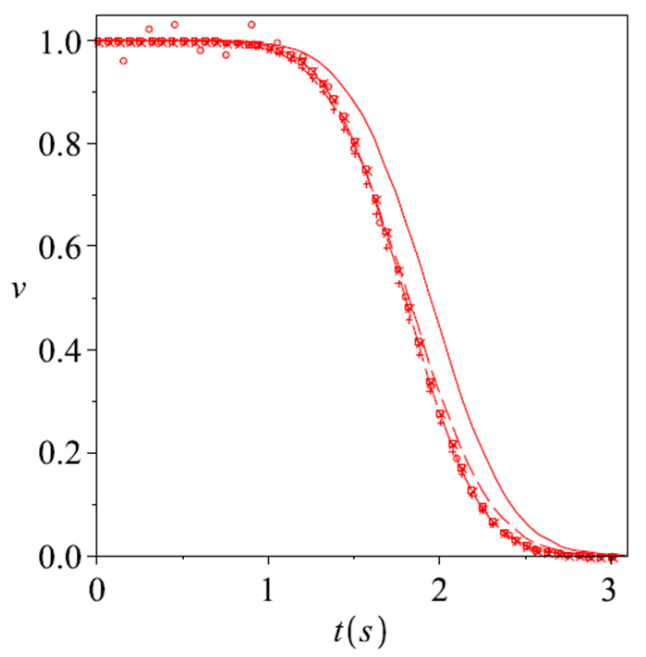

Figure 1: Comparison of normalized fractional yield not yet released $(v)$ vs time in seconds. The circles are data, the solid line is the initial guessed solution $\left(\theta^{(0)}\right)$, the dashed line is the result after one iteration, using parameters $\left(\theta^{(1)}\right)$, the diagonal crosses indicate the result after two iterations, with parameters $\left(\theta^{(2)}\right)$, the crosses indicate the result after three iterations, with parameters $\left(\theta^{(3)}\right)$, the boxes indicate the fourth result with parameters $\left(\theta^{(4)}\right)$ and the line composed of long dashes indicates the result after five iterations, with parameters $\left(\theta^{(5)}\right)$. These parameters are shown in Table 1.

to each other and it is difficult to see the fit improving. The improvement of fit is easier to see in a plot of the residuals as in Figure 2, the plot of residuals from Figure 1 with the same symbols.

\subsection{Digitised Data}

So far we have seen that even though two of the parameters to be fitted are highly correlated, the Levenberg-Marquardt method can be used successfully to fit $v^{(1)}$ to data generated using the leading asymptotic behaviour 


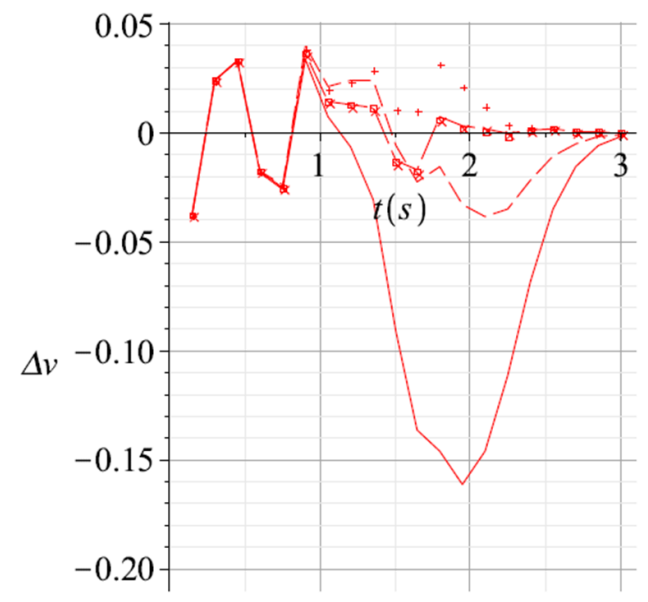

Figure 2: Residuals of the fits shown in Figure 1. Symbols used are the same.

of volatile released based on a Gaussian distribution, plus a small random additive error. We then tested the method using $v^{(1)}$ to fit data which has been digitised directly from published coal volatilisation experiments. Note that when working with digitised actual data the underlying distribution is unknown. Using digitized data demonstrates that this present method is promising for use with coal data with an unknown underlying distribution, even if the initial guess for the distribution $f_{0}$ is Gaussian. Again we use the Levenberg-Marquardt method, and we fit to actual coal data the leading order solution $v^{(1)}$ of Equation (31) based on a Gaussian distribution. We find that the present method works on this data, which has been digitized from published coal volatilization experiments [28]. Figure (3) illustrates the process, showing successive iterations converging to the data points, and Figure (4) shows the residuals of those fits.

\subsection{Higher-Order Correction}

We seek to improve our fit and further reduce residuals by adding the higherorder correction term $\epsilon f_{1}$ (which is non-Gaussian) to the leading-order Gaussian term $f_{0}$ of $v^{(1)}$ and repeating the iterative process shown above, by using the same digitized data [28]. Using the high-order approximation to $f,\left(f_{0}+\epsilon f_{1}\right)$ in the formula $v^{(1)}=\int_{y s}^{\infty} f(y) d y$ before fitting $v^{(1)}$ to data is one approach to obtaining a more accurate estimate of the underlying distribution in the DAEM.

The data is fitted with

$$
v_{1}^{(1)}=\int_{y_{s}}^{\infty}\left(f_{0}+\epsilon f_{1}\right) d y
$$




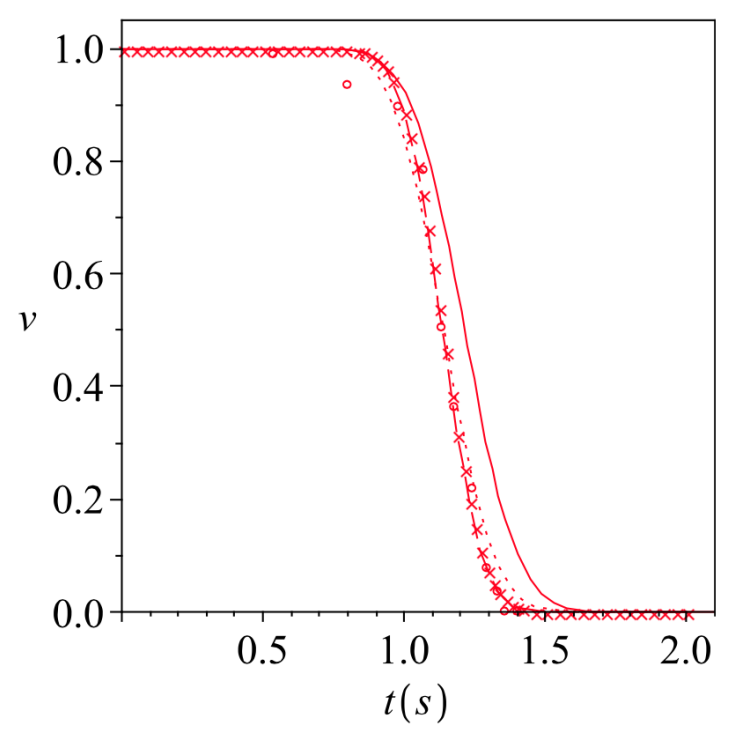

Figure 3: Data (circles) from a digitised graph of volatilisation measurements [28], showing total normalised amount of volatiles not yet released versus time, and successive fits to that data. The solid line is the initial guessed solution, the dotted line is the result after one iteration, the crosses indicate the result after two iterations, and the dashed line indicates the result after four iterations.

where

$$
f_{0}=-\frac{d v_{0}^{(1)} / d t}{d E_{s} / d t}
$$

and

$$
\epsilon f_{1}=A_{0}\left(E_{w} \frac{d f_{0}}{d E_{s}}+\frac{\frac{d E_{w}}{d t} f_{0}}{\frac{d E_{s}}{d t}}\right)
$$

so that

$$
v_{1}^{(1)}=\int_{y_{s}}^{\infty}\left(-\frac{d v_{0}^{(1)} / d t}{d E_{s} / d t}+A_{0}\left(E_{w} \frac{d f_{0}}{d E_{s}}+\frac{\frac{d E_{w}}{d t} f_{0}}{\frac{d E_{s}}{d t}}\right)\right) d y .
$$

We change the variable of integration in Equation (43) to the time $t$. Our approximation to the volatilisation is then

$$
v_{1}^{(1)} \sim \int_{t_{s}}^{\infty}\left(-\frac{d v_{0}^{(1)} / d t}{d E_{s} / d t}+A_{0}\left(E_{w} \frac{d f_{0}}{d E_{s}}+\frac{\frac{d E_{w}}{d t} f_{0}}{\frac{d E_{s}}{d t}}\right)\right) \frac{K}{E_{0}} d t
$$

where

$$
K=R m Y\left(k_{0} t\right)+\frac{R m Y\left(k_{0} t\right)}{1+Y\left(k_{0} t\right)},
$$




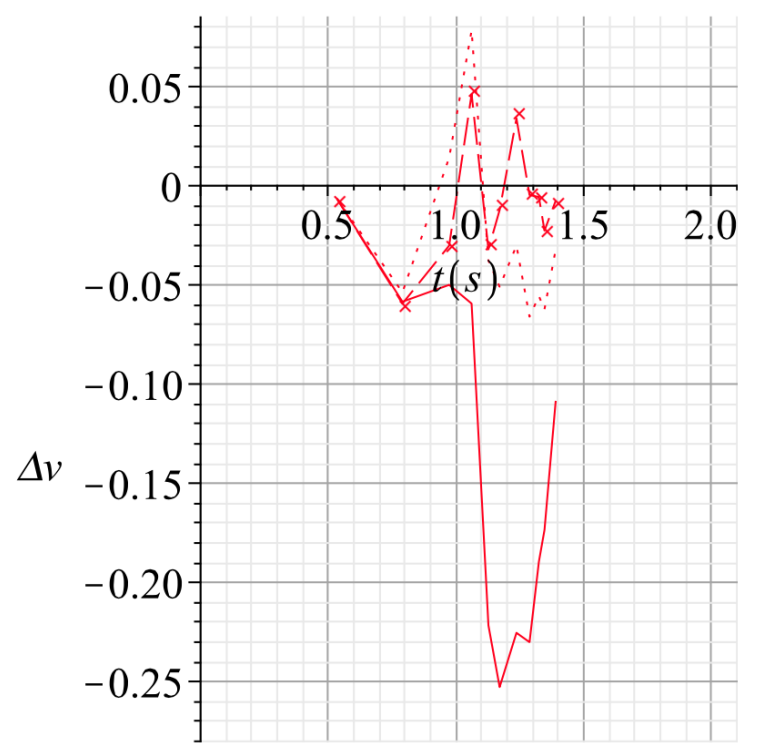

Figure 4: Residuals of the fits shown in Figure 3. Symbols used are the same.

and

$$
t_{s}=\sqrt{\frac{t}{k_{0} Y\left(k_{0} t\right)}} .
$$

Then $v_{1}^{(1)}$ is now rewritten as $v_{1}^{(1 *)}=1-v_{1}^{(1)}$ to give the fraction of the volatile yield not yet released,

$$
v_{1}^{(1 *)} \sim 1-\int_{t_{s}}^{\infty}\left(-\frac{d v_{0}^{(1)} / d t}{d E_{s} / d t}+A_{0}\left(E_{w} \frac{d f_{0}}{d E_{s}}+\frac{\frac{d E_{w}}{d t} f_{0}}{\frac{d E_{s}}{d t}}\right)\right) \frac{K}{E_{0}} d t
$$

where, differentiating Equation (38),

$$
\frac{d v_{0}^{(1)}}{d t}=-\frac{1}{\sqrt{2 \pi} \sigma} \exp \left[-\frac{\left(\operatorname{Rmt} Y\left(k_{0} t\right)-E_{0}\right)^{2}}{2 \sigma^{2}}\right] \operatorname{Rmt} Y\left(k_{0} t\right)\left(\frac{2+Y\left(k_{0} t\right)}{1+Y\left(k_{0} t\right)}\right) .
$$

We again use the Levenberg-Marquardt Algorithm procedure to fit the digitised data as described above. Note that the starting parameter estimates $\theta^{(0)}$ are chosen to be the same as the last parameter values that were obtained from the last (converged) iteration of $v^{(1)_{0}}$. To determine the convergence of the iterative process we used Equation (42). Figure (5) illustrates the process, showing successive iterations converging to the data points, and Figure (6) shows the residuals of those fits. 


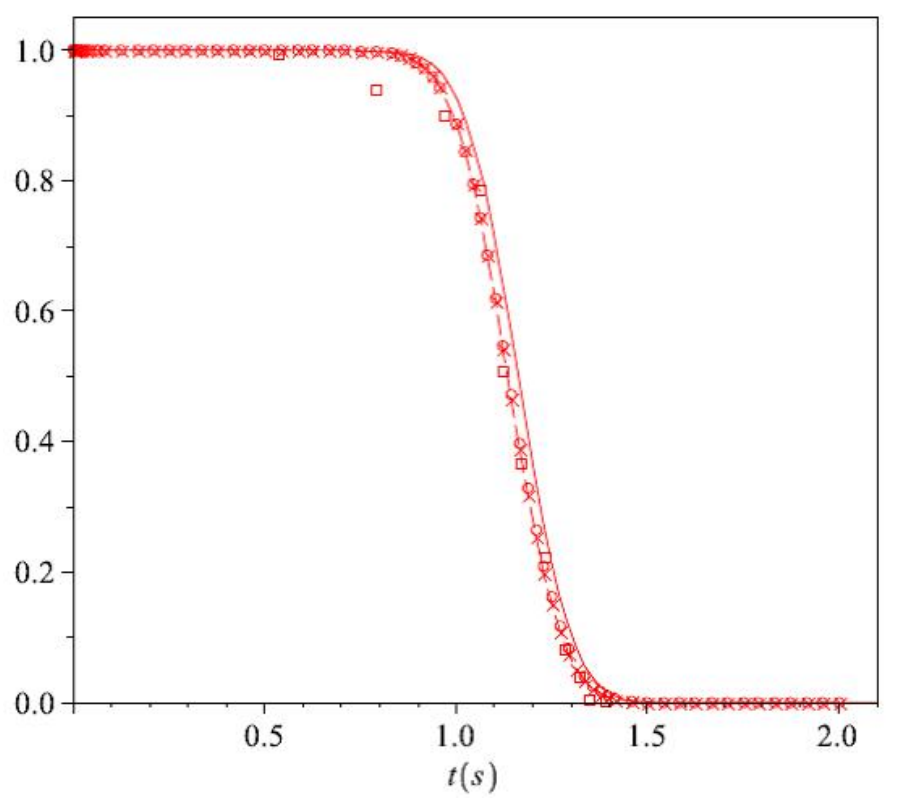

Figure 5: Data (boxes) from a digitised graph of volatilisation measurements [28], showing total normalised amount of volatiles not yet released versus time, and successive fits to that data. The solid line is the initial guessed solution, the circles are the result after one iteration, the crosses indicate the result after two iterations, and the dashed line indicates the result after third iterations.

\subsection{Comparison of $v_{0}^{(1)}$ and $v_{1}^{(1 *)}$}

Comparisons of the two fitted equations $v_{0}^{(1)}$ and $v_{1}^{(1 *)}$ illustrate that the results obtained by adding the higher-order correction term $\epsilon f_{1}$ to the leadingorder term $f_{0}$ provide an improved estimate of the underlying distribution. This can be seen both from the sums of squares and from plots of the residuals. For example after seven iterations, the results are illustrated in Table 2 below. The sum of squares in Table 2 illustrates that $v^{(1 *)_{1}}$ provides a more

\begin{tabular}{|r|c|c|}
\hline & $\boldsymbol{\theta}^{(7)}$ in $v_{0}^{(1)}$ & $\boldsymbol{\theta}^{(7)}$ in $v_{1}^{(1 *)}$ \\
\hline$S\left(\boldsymbol{\theta}^{(7)}\right)$ & 0.009596682368 & 0.009575375913 \\
$E_{0}$ & 222004.3158 & 217815.530752700288 \\
$\sigma$ & 22623.51229 & 22850.3934920922802 \\
$k_{0}$ & $8.52305343 \mathrm{E} 10$ & $9.68677057029868622 \mathrm{E} 10$ \\
\hline
\end{tabular}

Table 2: The parameters $\boldsymbol{\theta}^{(7)}$ after seven iterations in both fitting equations $v_{0}^{(1)}$ and $v_{1}^{(1 *)}$.

accurate estimate because the sum of squared residuals $S$ is smaller for the 


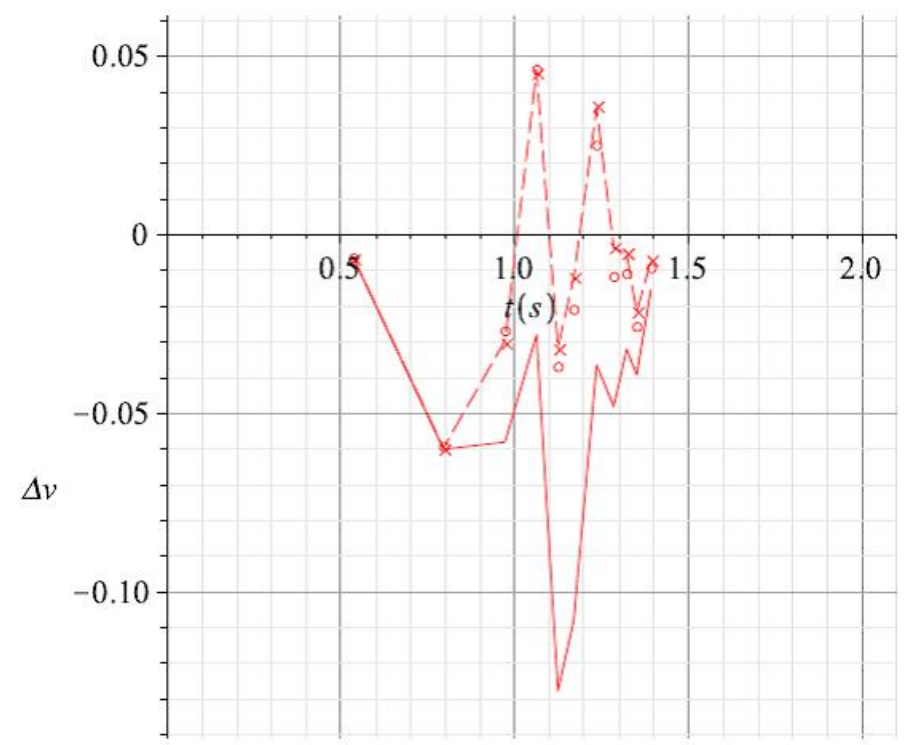

Figure 6: Residuals of the fits shown in Figure 5. Symbols used are the same.

latter. Note that both iterative processes showed convergence when $N=7$.

A plot of the residuals of these fits to digitised data versus time is provided in Figure 7 with the crosses being $v_{0}^{(1)}$ calculations, and the solid circles being those of $v^{(1 *)_{1}}$ calculations. It can be seen that the latter provide smaller residuals and a better fit. The second plot shows the residual results versus fitted values from Figure 7 with the $v^{(1)}$ results shown as crosses and $v^{(2 *)}$ results shown as solid circles. Figure 8 shows that the residuals are generally small compared to the fitted values and that they do not suggest any distinct pattern. All residuals in $v^{(1)}$ are typically less than 0.13 in absolute value, whereas the residuals in $v^{(2 *)}$ are less than 0.06 in absolute value. This suggests that the results from $v^{(2 *)}$ provides a more accurate estimate parameters than $v^{(1)}$. The analysis of the plotting in Figure 8 agrees with the chosen iterative method.

The inverse problem of determining the distribution from measurements of $v$ versus time when $\boldsymbol{\theta}=(217815.530752700288,22850.3934920922802$, 9.68677057029868622E10 is illustrated in Figure 9. Note that when working with digitized actual data, the distribution is unknown. The crosses in Figure 9 show the result of calculating just the leading-order term $f_{0}$ using Equation (34), and the solid circles are the result obtained by adding our higher-order correction term from Equation (35). It can be seen that adding $\epsilon f_{1}$ to $f_{0}$ shifts the underlying distribution a little bit toward the right which suggests that adding $\epsilon f_{1}$ gives a non Gaussian total distribution, that is, the actual data distribution is non-Gaussian otherwise, the best fit of $f_{0}$ would lie exactly under it. This result suggests that the method may be useful for 


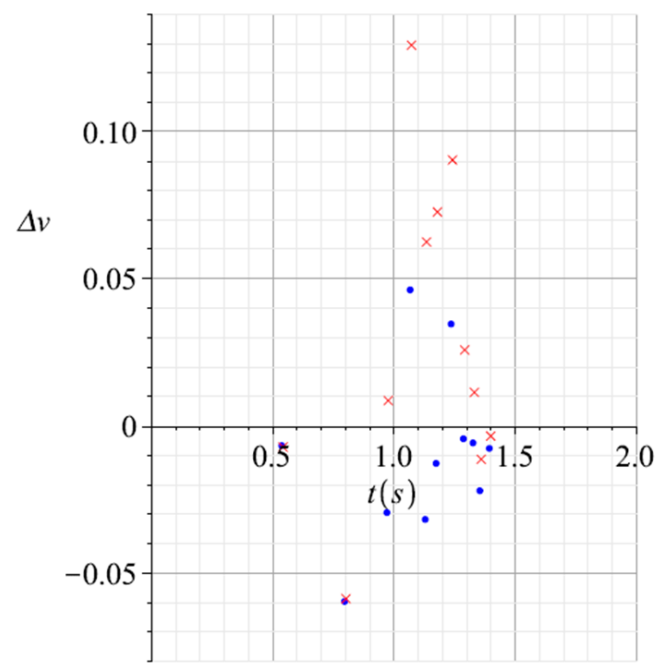

Figure 7: Comparison between residuals after fitting $v_{0}^{(1)}$ and $v_{1}^{(1 *)}$ to digitised data, at $\boldsymbol{\theta}=(217815.530752700288,22850.3934920922802$, $9.68677057029868622 \mathrm{E} 10)$. The crosses are $v_{0}^{(1)}$ calculations, and the solid circles are of $v_{1}^{(1 *)}$ calculations.

fitting non Gaussian underlying distributions. The underlying distribution is smooth and shown in Figure 9.

Note that during the iterative process, special attention needs to be paid to how to increase and decrease the conditioning factor $k^{(n)}$ that is used in the Levenberg-Marquardt method. There are two approaches to dealing with the situation that may arise, that at some iteration the new sum of squares is greater than the previous one. The first approach is to increase $k^{(n)}$ by a factor and use this together with the most recent (increased sum of squares) parameter values to perform the next iteration. The second approach is to increase $k^{(n)}$ by a factor and use this together with the last decreased sum of squares parameter values to compute the next iteration. We find that the second approach converges faster than the first approach.

\section{Conclusions}

The DAEM model for the amount of volatile mass released versus time during coal volatilisation has an exact solution. However, evaluation of this solution involves a double integral and a double exponential function, making it expensive to repeatedly evaluate. We have reviewed and extended previous work, to approximate the solution without expensive multiple evaluations by using a succession of asymptotic approximations, in the case of a relatively wide distribution function that describes the way that volatility 


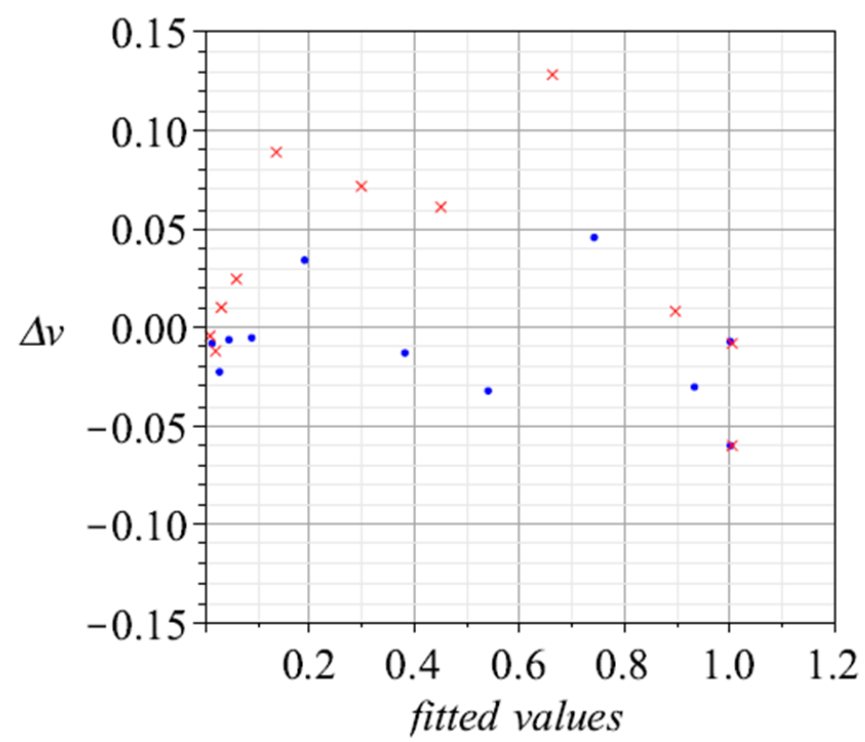

Figure 8: A plot of residuals versus fitted values of $v_{0}^{(1)}$ and $v_{1}^{(1 *)}$ at $\boldsymbol{\theta}=$ (217815.530752700288, 22850.3934920922802, 9.68677057029868622E10) from Figure 7. The crosses are $v_{0}^{(1)}$ calculations, and the solid circles are $v_{1}^{(1 *)}$ calculations.

depends on the energy.

In particular we have considered the use of these asymptotic expansions for solving the inverse problem - given the mass of volatile released versus time, find the underlying relatively wide distribution of volatiles versus energy. Direct differencing of the volatility data leads to ever increasing errors which can swamp the signal with noise, and prevent taking more accurate approximations which require higher order differences.

We have outlined and tested a method for smoothing the volatility data, using the leading-order Gaussian approximation to allow higher derivatives to be taken without incurring the differencing error penalty. We find that the method is promising, even in situations where the underlying distribution may not be Gaussian.

We have two expansion directions, one that approximates the total mass of volatile released,

$$
\begin{aligned}
v \sim & v^{(1)}+v^{(2)}+\ldots \\
\sim & \int_{y s}^{\infty} f(y) d y+y_{w} A_{0} f\left(y_{s}\right)+y_{w}^{2} A_{1} f^{\prime}\left(y_{s}\right) \\
& +y_{w}^{3} A_{2} f^{\prime \prime}\left(y_{s}\right) / 2+y_{w}^{4} A_{3} f^{\prime \prime \prime}\left(y_{s}\right) / 6+ \\
& y_{w}^{5} A_{4} f^{i v}\left(y_{s}\right) / 24+y_{w}^{6} A_{5} f^{v}\left(y_{s}\right) / 120 .
\end{aligned}
$$






Figure 9: Comparison of the underlying distributions estimated inversely from digitised values of $v$ versus time for the leading-order result and the higher-order result. The leading-order result using $v_{0}^{(1)}$ is represented by crosses, and the higher-order result using $v_{1}^{(1 *)}$ is represented by solid circles.

and another that approximates the underlying distribution,

$$
\begin{aligned}
f \sim & f_{0}+\epsilon f_{1}+\epsilon^{2} f_{2}+\ldots \\
\sim & -\frac{d v / d t}{d E_{s} / d t}+A_{0}\left(E_{w} \frac{d f_{0}}{d E_{s}}+\frac{\frac{d E_{w}}{d t} f_{0}}{\frac{d E_{s}}{d t}}\right) \\
& +\epsilon A_{0}\left(\frac{\frac{d E_{w}}{d t} f_{1}}{\frac{d E_{s}}{d t}}+E_{w} f_{1}^{\prime}\right)+\ldots
\end{aligned}
$$

We explored the direction of using $f_{0}$ and $f_{0}+\epsilon f_{1}$ to approximate $v^{(1)}$ which approximates $v$ in this paper. We have not yet explored the other direction, of improving the approximation to $v$ by using more terms $v^{(1)}+v^{(2)}+$ .... Also useful for the future would be to explore which direction is more important, that is, the relative sizes of corrections in the two directions.

\section{References}

[1] J. Howard and M. Elliott, "Chemistry of coal utilization," 2nd Suppl. Vol., chap 12, 1981. 
[2] P. Solomon, D. Hamblen, and R. Carangelo, "Coal pyrolysis, aiche," in Symposium on Coal Pyrolysis (Nov., 1981). Solomon, PR and Colket, MB, Fuel, vol. 7, p. 16, 1978.

[3] S. Niksa and C.-W. Lau, "Global rates of devolatilization for various coal types," Combustion and flame, vol. 94, no. 3, pp. 293-307, 1993.

[4] E. Suuberg, "Approximate solution technique for nonisothermal, gaussian distributed activation energy models," Combustion and Flame, vol. 50, pp. 243-245, 1983.

[5] K. Miura and T. Maki, "Simplified method to estimate f (e) in distributed activation energy model for analyzing coal pyrolysis reaction," Journal of chemical engineering of Japan, vol. 31, no. 2, pp. 228-235, 1998.

[6] C. Please, M. McGuinness, and D. McElwain, "Approximations to the distributed activation energy model for the pyrolysis of coal," Combustion and Flame, vol. 133, no. 1, pp. 107-117, 2003.

[7] B. Urych, "Determination of kinetic parameters of coal pyrolysis to simulate the process of underground gasification (ucg)," Journal of sustainable mining, vol. 13, pp. 3-9, 2014.

[8] V. Vand, "A theory of the irreversible electrical resistance changes of metallic films evaporated in vacuum," Proceedings of the Physical Society, vol. 55, no. 3, p. 222, 1943.

[9] G. Pitt, "The kinetics of the evolution of volatile products from coal," Fuel, vol. 41, no. 3, pp. 267-274, 1962.

[10] K. Miura, "A new and simple method to estimate $f(e)$ and $k_{0}(e)$ in the distributed activation energy model from three sets of experimental data," Energy \& Fuels, vol. 9, no. 2, pp. 302-307, 1995.

[11] T. Maki, A. Takatsuno, and K. Miura, "Analysis of pyrolysis reactions of various coals including argonne premium coals using a new distributed activation energy model," Energy \& Fuels, vol. 11, no. 5, pp. 972-977, 1997.

[12] P. R. Solomon and D. G. Hamblen, "Finding order in coal pyrolysis kinetics," Progress in energy and combustion science, vol. 9, no. 4, pp. 323-361, 1983.

[13] D. B. Anthony and J. B. Howard, "Coal devolatilization and hydrogastification," AIChE Journal, vol. 22, no. 4, pp. 625-656, 1976. 
[14] H. Juntgen, "Review of the kinetics of pyrolysis and hydro-pyrolysis in relation to the chemical constitution of coal," Fuel, vol. 63, pp. 731-737, 1983.

[15] J. Howard and R. Essenhigh, "Pyrolysis of coal particles in pulverized fuel flames," Industrial 83 Engineering Chemistry Process Design and Development, vol. 6, no. 1, pp. 74-84, 1967.

[16] S. Badzioch and P. G. Hawksley, "Kinetics of thermal decomposition of pulverized coal particles," Industrial \& Engineering Chemistry Process Design and Development, vol. 9, no. 4, pp. 521-530, 1970.

[17] S. Saxena, "Devolatilization and combustion characteristics of coal particles," Progress in energy and combustion science, vol. 16, no. 1, pp. 5594, 1990.

[18] D. Anthony, J. Howard, H. Hottel, and H. Meissner, "Rapid devolatilization of pulverized coal," in Symposium (International) on Combustion, vol. 15, pp. 1303-1317, Elsevier, 1975.

[19] P. R. Solomon, M. A. Serio, and E. M. Suuberg, "Coal pyrolysis: experiments, kinetic rates and mechanisms," Progress in Energy and Combustion Science, vol. 18, no. 2, pp. 133-220, 1992.

[20] M. Gunes and S. Gunes, "The influences of various parameters on the numerical solution of nonisothermal daem equation," Thermochimica Acta, vol. 336, pp. 93-96, 1999.

[21] M. E. Brown, "Introduction to thermal analysis: Techniques and applications. 1988."

[22] H. Teng and C. Hsieh, "Activation energy for oxygen chemisorption on carbon at low temperatures," Journal of sustainable mining, vol. 38, pp. 292-297, 1999.

[23] C. Lakshmanan, M. Bennett, and N. White, "Implications of multiplicity in kinetic parameters to petroleum exploration: Distributed activation energy models," Energy \& Fuels, vol. 51, pp. 110-117, 1991.

[24] D. Merrick, "Mathematical models of the thermal decomposition of coal. the evolution of volatile matter," Fuel, vol. 62, pp. 534-539, 1983.

[25] E. Donskoi and M. D.L.S, "Approximate modelling of coal pyrolysis," Fuel, vol. 78, pp. 825-835, 1999.

[26] R. Armstrong and B. Kulesza, "An approximate solution to the equation $\mathrm{x}=\exp (-\mathrm{x} / \epsilon)$," Bull. Institute of Mathematics and its Applications, vol. 17, p. 56, 1981. 
[27] G. Seber and C. Wild, "Nonlinear regression. 2003."

[28] X. MA, H. Nagaishi, T. Yoshida, G. Xu, and M. Harada, "Kinetics of rapid coal devolatilization measured using a spot heater apparatus," Fuel processing technology, vol. 85, pp. 43-49, 2004. 